\title{
Problemas de hierarquização espacial e funcional na ecologia da paisagem: uma avaliação a partir da abordagem geossistêmica
}

Lucas Costa de Souza Cavalcanti* Antonio Carlos de Barros Corrêa

\section{Resumo}

Em seu contexto físico, o zoneamento ambiental está imerso numa profunda e antiga discussão envolvendo divergências terminológicas e conceituais. Neste trabalho buscou-se esclarecer aspectos desta discussão com base na Teoria Hierárquica (TH). Foram identificadas categorias comuns da hierarquia ambiental e as diferentes nomenclaturas utilizadas. Em seguida são apresentados aspectos gerais da TH e são sumarizados conceitos para o entendimento de hierarquias escalares. É discutida a relação entre os conceitos de ecossistema e geossistema; são tratadas algumas das abordagens contemporâneas para identificação de unidades hierárquicas do ambiente. Enfim, verificam-se a diferença e complementaridade entre os conceitos de geossistema e ecossistema; a aplicabilidade de modelos tradicionais e o desafio na representação da dinâmica dos geossistemas.

Palavras-chave: Teoria Hierárquica; Ecologia da Paisagem; Hierarquia Ambiental.

* Professor da Universidade de Pernambuco/Campus III - Petrolina, é pesquisador do Grupo de Pesquisa em Sociedade e Natureza do Vale do São Francisco e membro do Grupo de Estudos do Quaternário do Nordeste Brasileiro (lucascavalcanti3@gmail.com).

** Doutor em Geografia pela Universidade Estadual Paulista Júlio de Mesquita Filho com estágio sanduiche na Universidade de Durham, Reino Unido e pós-doutorado em geomorfologia pela Universidade Estadual Paulista Júlio de Mesquita Filho (dbiase2001 @ terra.com).

Geosul, Florianópolis, v. 28, n. 55, p 143-162, jan./jun. 2013 
CAVALCANTI, L.C.S. \& CORRÊA, A.C.B. Problemas de hierarquização...

Spatial and functional hierarchization problems in landscape ecology: an assessment based on the geosystems approach

\section{Abstract}

Within its physical context, environmental zoning is immersed in a deep and long standing discussion involving terminological and conceptual divergences. This works aims at clarifying some aspects of this discussion based on Hierarchic Theory (HT). Common categories of environmental hierarchy were identified as well as the different nomenclature used. Following, general aspects of HT were presented and concepts were summarized for the understanding of scale hierarchies. Relation between the concepts of ecosystems and geosystems was discussed, as well as some contemporary approaches for the identification of environmental hierarchic units were put forward. At last, the differences and convergences between the concepts of geosystems and ecosystem were validated, likewise the applicability of traditional models and the challenge to represent geosystems dynamics.

Key words: Hierarchy Theory; Landscape Ecology; Environmental Hierarchy.

\section{Introdução}

$\mathrm{O}$ zoneamento ambiental é uma ferramenta fundamental do planejamento territorial. Conhecer a tipologia de unidades ambientais é necessário para melhor compreender as possíveis respostas de tais unidades, seja às pressões antrópicas, às mudanças climáticas globais ou eventos perturbadores de grande magnitude. Reconhecer as unidades ambientais (enquanto indivíduos geográficos) permite realizar inferências sobre suas relações espaciais, sobretudo as relações escalares (hierárquicas), viabilizando simulações sobre seu futuro e seu passado. Todavia, o estudo de unidades ambientais para zoneamento possui um contexto de profunda discussão relativo aos critérios de sua 
CAVALCANTI, L.C.S. \& CORRÊA, A.C.B. Problemas de hierarquização... definição e mesmo sua posição no contexto epistemológico da Ecologia da Paisagem.

No âmbito da cartografia de geossistemas, o reconhecido modelo hierárquico de Bertrand (1972): "geossistemas-geofáciesgeótopos" tem recebido muitas críticas, sobretudo pelo fato de associar a cada uma das unidades uma dimensão espacial (ordem de grandeza) definida, que pode encontrar aplicabilidade para o território francês, mas que tem sido aplicado com dificuldade ao contexto brasileiro, como já percebido por diversos autores (MONTEIRO, 1976; AB'SABER, 2007; MELO, 2008).

O objetivo deste trabalho é ampliar a discussão sobre a classificação hierárquica de unidades ambientais para fins de cartografia de síntese, sobretudo pela investigação de elementos gerais ás classificações deste tipo e apontar alguns de seus principais problemas. Aqui vamos enfocar um modelo hierárquico alternativo, muito comum na geografia russo-soviética e que fundamenta as propostas taxonômicas de autores como Sochava e Isachenko.

\section{Hierarquia de unidades ambientais e sua classificação}

Neste trabalho as paisagens são consideradas como sistemas complexos e hierárquicos. Assim, a análise das principais ideias e termos relacionados à análise ambiental baseou-se nos fundamentos da Teoria Hierárquica, construída como parte da Teoria dos Sistemas por pesquisadores de diversas áreas (Economia, Química, Biologia, Psicologia, etc.) (AHL \& ALLEN, 1996) e com forte presença nos trabalhos de alguns geógrafos (SOCHAVA, 1977).

O reconhecimento de uma organização hierárquica para o funcionamento dos processos naturais é antiga. Em resumo podemse destacar três momentos históricos fundamentais para compreensão da paisagem enquanto hierarquia. O primeiro deles é influenciado pelas idéias holísticas, organicistas, da Filosofia da 
CAVALCANTI, L.C.S. \& CORRÊA, A.C.B. Problemas de hierarquização...

Natureza entre os séculos 18, 19 e início do século 20. Neste momento o ambiente é visto como um organismo.

Em termos de hierarquia propõem-se os modelos para análise de grandes dimensões territoriais, através dos conceitos de zonalidade e azonalidade. Destacam-se os seguintes trabalhos: "Teoria das Zonas Naturais" de Vasiliy Vasilievich Dokuchaev, publicado em 1892; "As principais regiões naturais: um ensaio de geografia sistemática” de A. J. Herbertson, publicado em 1905; "Objeto e problemas da Geografia" (1915) e "Zonas de paisagens-geográficas da URSS” (1930) ambos de Lev Semionovich Berg; Além de diversos artigos escritos por Sigfried Passarge ainda na década de 1910 (ISACHENKO, 1973).

A partir da década de 20, o progresso no Landscape Surveying conduziu à necessidade de estabelecimento de uma hierarquia para as unidades ambientais (ISACHENKO, 1973). Logo, apareceram modelos que continham unidades espaciais elementares (indivisíveis), diferenciadas pela relação solos, rochas, relevo e vegetação. Procedimentos similares ocorreram em outros países, como a Alemanha e Estados Unidos (MASSEY apud ISACHENKO, 1973). Estas unidades mínimas são reconhecidas como sendo a menor associação funcional entre elementos ambientais (sobretudo relevo, solo, vegetação e rochas), tendo sido chamadas de Paisagens elementares (Boris Polinov e Krasheninnikov). Micro-paisagens (Larin), Epimorfos (Abolin), Elementos da terra ou Componentes da terra (Brink), Fácies (Ramenski, Solntcev e outros), Ecossistemas ou Sistemas ecológicos (Woltereck, Tansley e por quase todo mundo no ocidente, depois deles), Células da paisagem (Paffen), Ecótopos (Neef), Geótopos (Bertrand, Bolós e Haase), Elementos da paisagem (Kremsa), Geofácies (Sochava, Beruchashvili e Bertrand e os seguidores da teoria dos geossistemas); Geocomplexos elementares (Isachenko), Minibiomas (Ab'Sáber) Biogeocenoses (Sukachev), Plataformas (Schimidthusen), Geômeros elementares (Sochava), Micrócoro (Zonneveld), Elementos da Paisagem (Goosen), Sítio (Bailey), Ecoelemento (Klijn e Haes), Unidade de 
CAVALCANTI, L.C.S. \& CORRÊA, A.C.B. Problemas de hierarquização...

Terra/land unit (Bourne, Chrstian e a Food and Agriculture Organization of the United Nations - FAO).

A pedra fundamental para determinação da hierarquia da paisagem pode ser encontrada em dois artigos publicados por Nicolai Adolfovich Solntcev em 1948 e 1949, onde, a partir de longos trabalhos de campo, foram detalhados os níveis da organização ambiental. Solntcev (2006) propôs a existência de cinco níveis ${ }^{1}$ : Fácies; Subtratos (associação de Fácies), Tratos (associação de Fácies e/ou Subtratos - figura 1); Localidades (associação de Tratos - figura 2) e Paisagens (associação de Tratos e/ou Localidades - figura 3).

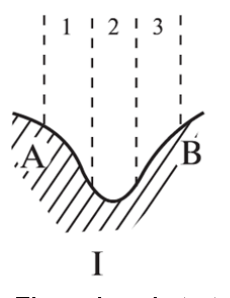

Tipos de substrato

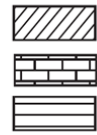

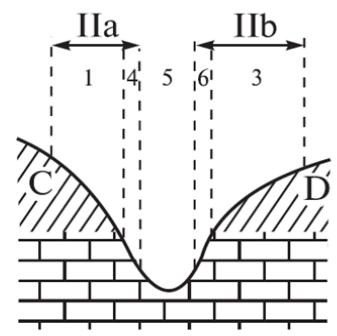

II

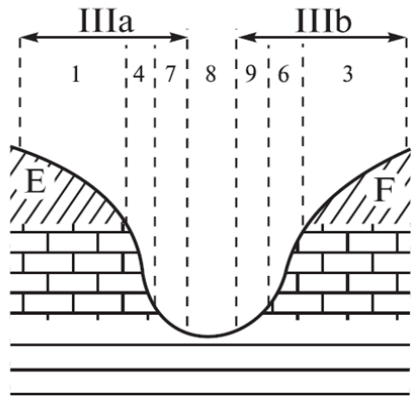

III

Figura 1: Estrutura dos Tratos. I Trato simples; II e III Trato complexo; IIa, IIb, IIIa e IIIb Subtrato. 1 a 9 Fácies. Modificado de Zuchkova \& Rakovskaia, 2004, p.33.

${ }^{1}$ Para correlação conceitual com as terminologias de outros autores ver: Kremsa, 2001. 
CAVALCANTI, L.C.S. \& CORRÊA, A.C.B. Problemas de hierarquização...

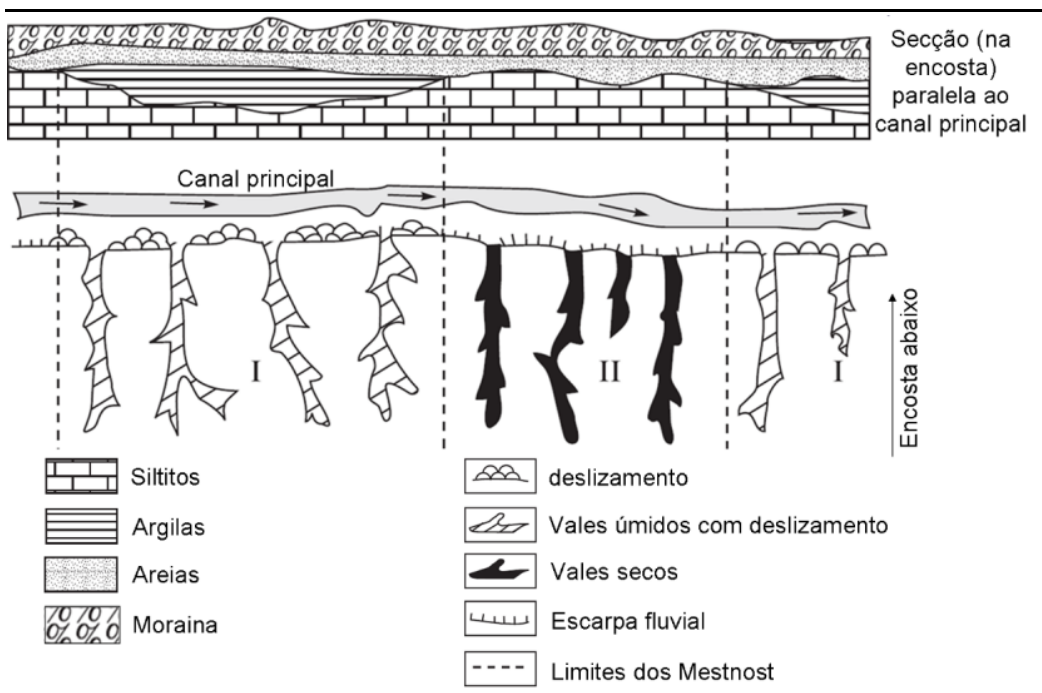

Figura 2: Esquema de diferenciação de uma Localidade (Mestnost) como uma unidade morfológica de uma paisagem. I - Localidade com Tratos em vales úmidos; II - Localidade com Tratos em vales secos. Fonte: Modificado de Zuchkova \& Rakovskaia, 2004, p.36.

Um segundo momento dos estudos da hierarquia das paisagens apareceu com a incorporação do paradigma da teoria dos sistemas.

O advento dos esquemas em hierarquia permitiu grande avanço para o planejamento territorial. Todavia, a aplicação destes esquemas considerava apenas aspectos morfológicos e genéticos da paisagem. Pouco se estudava sobre sua dinâmica, isto é, sobre seu funcionamento e evolução. Esta característica pode ser atribuída à fundamentação essencialmente organicista dos conceitos. Para Mamay (2007) unicamente após a incorporação do paradigma sistêmico é que se tornou possível, para os estudos ambientais, representar a dimensão dinâmica da natureza. 
CAVALCANTI, L.C.S. \& CORRÊA, A.C.B. Problemas de hierarquização...

No âmbito dos estudos integrados do ambiente, atribui-se a Viktor Borisovich Sochava (1905-1978) a incorporação do paradigma sistêmico ao estudo dos complexos naturais integrados. Sochava considera o ambiente como um sistema contínuo de fluxos de substâncias e, ainda na década de 1960, lidera o estabelecimento das primeiras estações de monitoramento da paisagem como um todo, com o objetivo de acompanhar os processos ambientais e elaborar modelos preditivos. Dentre as estações de monitoramento, a que se tornou mais famosa foi a Estação Físico-Geográfica de Martkopi, na Geórgia, que, sob orientação de Nikolai Levanovich Beruchashvili, vem realizando, há cerca de 40 anos, medidas diárias em 100 variáveis na paisagem para fins de modelagem ambiental, obtendo entre 5000 a 6000 medidas diárias. (BERUCHASHVILI, 2007).

Os estudos em estações vieram a destacar o fato da relatividade no mapeamento ambiental. Esta relatividade deriva do fato de que os padrões geográficos (espaciais) dos sistemas da natureza dependem largamente do modelo de componentes utilizado (KRUHLOV, 1999; KRUHLOV \& BOZHUK, 2004). Como os modelos tradicionais utilizam dados de cobertura vegetal, relevo, rochas e solos os resultados sempre são semelhantes. Todavia, alterando-se os parâmetros, os limites geográficos das unidades podem ser completamente diferentes. Por exemplo, no caso de se considerar variações na umidade do ar como um parâmetro os limites dos geossistemas irão se alterar rapidamente. Os modelos de componentes (também chamados geocomponentes) podem ser classificados conforme sua variabilidade no tempo, sua mobilidade espacial e sua habilidade para complicar sua estrutura (KRAUKLIS, 1979). 
CAVALCANTI, L.C.S. \& CORRÊA, A.C.B. Problemas de hierarquização...

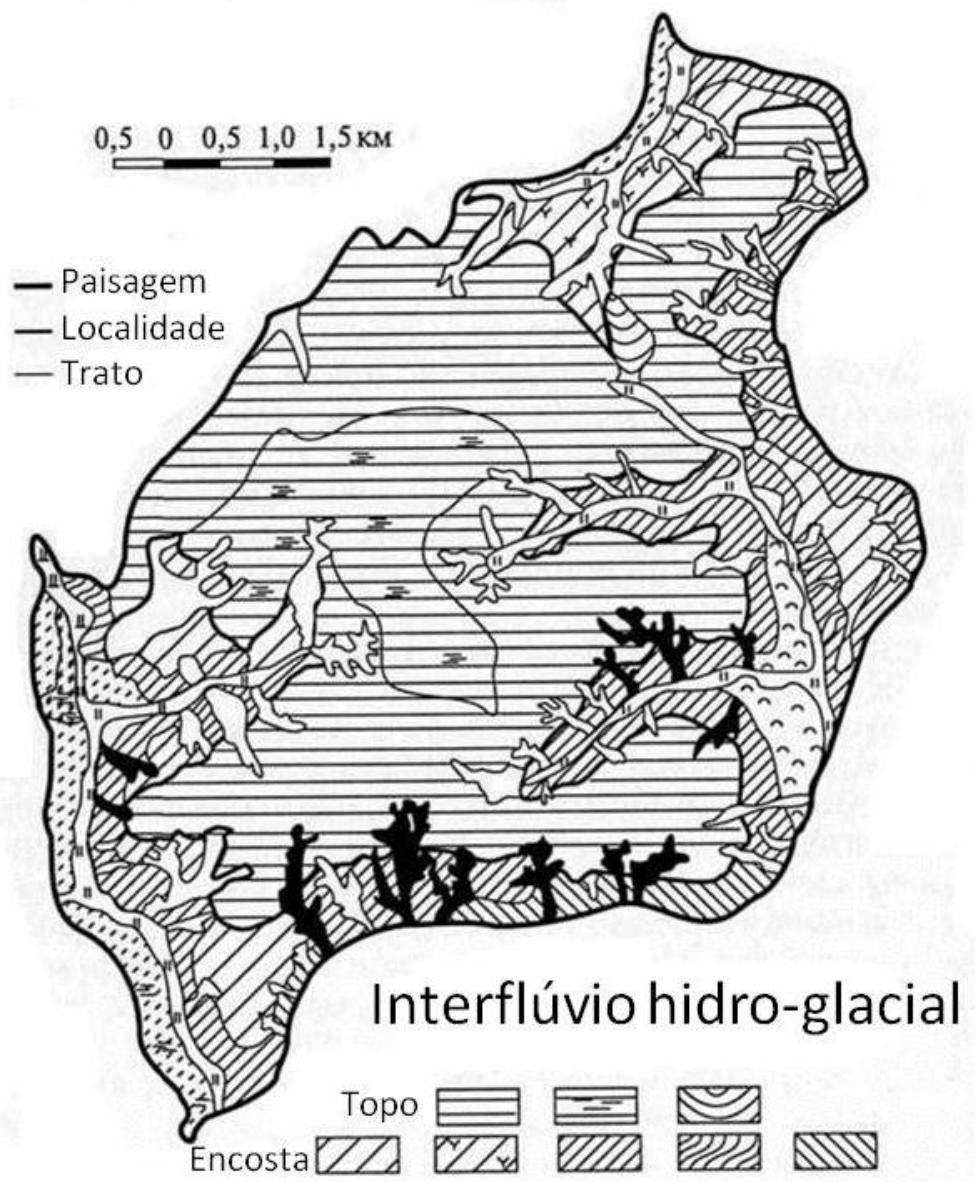

Terraçose

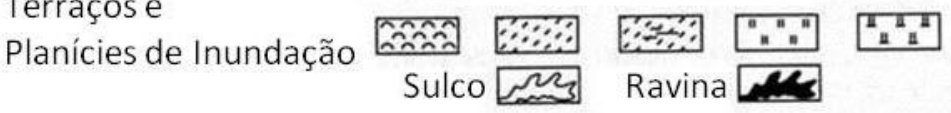

Figura 2: Paisagem interfluvial na margem direita do Rio Iput (cerca de $500 \mathrm{~km}$ a SW de Moscou). Fonte: Modificado de Zuchkova \& Rakovskaia, 2004, p.38. 
CAVALCANTI, L.C.S. \& CORRÊA, A.C.B. Problemas de hierarquização...

Quanto à sua variabilidade temporal os modelos podem ser estacionários (estáticos), ex: depósitos geológicos, estruturas arquitetônicas; ou circulativos (dinâmicos), ex: massas de ar, vegetação, solos (KRAUKLIS, 1979.). Os componentes estacionários também são denominados de Sítios (ISACHENKO, 2007) e Abióticos (KHOROSHEV \& MEREKALOVA, 2006). Os componentes circulativos são também denominados de Móveis (ISACHENKO, 2007; KHOROSHEV \& MEREKALOVA, 2006). Quanto à sua mobilidade espacial podem ser migratórios (massas de ar, populações humanas) ou residentes (vegetação, estruturas arquitetônicas) (KRAUKLIS, 1979). Quanto à sua capacidade de complicar sua estrutura pode-se distinguir geocomponentes passivos (geocomponentes não vivos) ou ativos (geocomponentes bióticos, incluindo populações humanas) (KRAUKLIS, 1979).

Em geral, o modelo de geocomponentes mais utilizado se baseia nas relações entre relevo, solo/litotipo e vegetação (ZONNEVELD, 1989). Em muitos estudos busca-se identificar correlações entre os padrões espaciais dos geocomponentes através de métodos estatísticos (KHOROSHEV \& MEREKALOVA, 2006; PUEYO, et.al., 2007; SOLON, DEGÓRSKI \& ROO-ZIELIŃSKA, 2007; XU et.al., 2008).

O terceiro momento para compreensão dos estudos da paisagem como sistema hierárquico surge após o advento dos sistemas de informação geográfica e do sensoriamento remoto baseado em imagens de alta resolução. Todavia, este momento só pode ser compreendido a partir de alguns conceitos fundamentais enunciados a seguir.

\section{Hierarquia e escala: conceitos auxiliares}

Consideradas enquanto sistemas complexos, as paisagens apresentam uma estrutura hierárquica cujas relações são definidas em níveis de organização que possuem relações mais fortes no interior dos níveis do que entre níveis, além disso, cada nível opera numa escala espacial e temporal distinta (SIMON, 1962; HAY \& 
CAVALCANTI, L.C.S. \& CORRÊA, A.C.B. Problemas de hierarquização...

MARCEAU, 2004). A escala é fundamental para a concretização da hierarquia e da organização das paisagens (LEVIN, 1992; HAY \& MARCEAU, 2004).

Escala é definida como a dimensão espacial em que os padrões geográficos são observados e medidos (MARCEAU, 1999). A escala pode ser absoluta ou relativa. A escala absoluta corresponde ao sistema padrão para dividir o espaço geográfico em unidades operacionais de estudo, como a escala cartográfica e/ou unidades censitárias (HAY \& MARCEAU, 2004). A escala relativa diz respeito à definição dos elementos espaciais uns em relação aos outros (MARCEAU, 1999).

A escala é formada por duas componentes: o grão e a extensão. O grão é o menor nível de resolução espacial numa dada observação (como o pixel de uma imagem) (SOARES FILHO, 1998). A extensão é o tamanho da área de estudo e o intervalo de tempo considerado (SOARES FILHO, 1998). A partir dessas definições uma pequena escala refere-se a uma área pequena, diferentemente do que ocorre com a escala cartográfica, o mesmo é válido para grande escala, referindo-se a uma área grande (HAY \& MARCEAU, 2004). Neste sentido, este trabalho diferencia escala geográfica (na instância de análise do fenômeno) de escala cartográfica (na instância de representação do fenômeno). No âmbito temporal, o grão corresponde a um instante observado, enquanto que a extensão é o conjunto de instantes observados (AHL \& ALLEN, 1996).

Os dois principais desafios para análise da escala em Geografia são chamados de problema da escala e do escalante (HAY \& MARCEAU, 2004).

O problema da escala trata da identificação de quais escalas são adequadas ao estudo e representação de determinados padrões e processos (MARCEAU, 1999). Algumas considerações importantes para resolução deste problema envolvem os conceitos de domínio de escala e limiar de escala (MARCEAU, 1999; HAY \& MARCEAU, 2004). O domínio de escala refere-se à continuidade de manifestação de padrões espaciais e processos, 
CAVALCANTI, L.C.S. \& CORRÊA, A.C.B. Problemas de hierarquização... isto significa que para uma determinada escala o fenômeno em questão não muda ou muda lentamente (MARCEAU, 1999; HAY \& MARCEAU, 2004). O limiar de escala corresponde ao ponto onde as mudanças nos padrões da paisagem ocorrem, ele define o fim de uma escala e um início de outra (MARCEAU, 1999; HAY \& MARCEAU, 2004).

O problema do escalante relaciona-se à definição automática de domínios de escala em ambiente computacional. Sua resolução requer a obtenção de informações acerca de processos e padrões em diversas amplitudes escalares para detectar limiares de escala e definir regras para transferência da informação através de múltiplas escalas (HAY \& MARCEAU, 2004).

Percebe-se que a análise hierárquica da paisagem depende de uma análise multiescalar. Neste sentido, é preciso ressaltar que uma análise em várias escalas pode seguir dois caminhos: direto ou indireto.

Uma aproximação multiescalar direta busca detectar os padrões dominantes e como eles surgem em escalas específicas a partir de um único conjunto de dados. Este tipo de abordagem inclui análise fractal, a teoria do espaço-escala, análise multiescala orientada ao objeto, entre outras técnicas (HAY \& MARCEAU, 2004).

Uma aproximação multiescalar indireta busca detectar os padrões a partir de dados adquiridos ou reamostrados para uma série de escalas discretas escolhidas arbitrariamente. A escolha arbitrária é a principal limitação desta abordagem, que ignora a continuidade da hierarquia, podendo gerar erros na detecção dos domínios de escala (HAY \& MARCEAU, 2004).

As análises realizadas em multiescala ainda podem ser classificadas quanto ao sentido da detecção da hierarquia. Partindose de grande escala geográfica para as escalas menores tem-se uma abordagem downscaling ou top-down. Partindo-se de pequenas escalas geográficas para escalas maiores tem-se uma abordagem upscaling ou bottom-up. 
CAVALCANTI, L.C.S. \& CORRÊA, A.C.B. Problemas de hierarquização...

\section{Identificação da hierarquia ambiental: problemas e perspectivas}

A análise hierárquica é necessária para compreensão do funcionamento dos sistemas ambientais. Neste sentido, as abordagens para análise ambiental podem ser classificadas de acordo com os princípios apresentados no item anterior. Tais abordagens são amplamente variadas e com potencial diversificado de aplicação, todavia, nenhuma delas está livre de problemas.

Reavaliando os modelos tradicionais, Khoroshev, Merekalova \& Aleschenko (2007) afirmam que os resultados obtidos com a aplicação de tecnologias computacionais para identificação de grande diversidade de ambientes têm demonstrado desacordo com os modelos tradicionais: nem sempre a cobertura vegetal responde a contraste de solos ou ao limite dos tratos; Muitas vezes uma fácies não pode ser explicada pelas propriedades do relevo ou litotipo. Os mesmos autores afirmam que o acúmulo de dados sobre estudos da paisagem tem demonstrado que os conceitos clássicos precisam ser considerados como casos particulares de uma regularidade que é na verdade, multiestrutural (KHOROSHEV, MEREKALOVA \& ALESCHENKO, 2007). Esta crítica é essencialmente metodológica, ou seja, as técnicas para mapeamento ambiental deveriam considerar a funcionalidade multiestrutural (de cada geocomponente), que não é o caso dos métodos tradicionais, que muitas vezes baseiam-se, grosso modo, no litotipo, declive e orientação da vertente, estrutura da vegetação e vegetação potencial.

A ideia de organização multiestrutural está essencialmente associada à funcionalidade dos geocomponentes e sempre esteve presente na análise ambiental, todavia, os métodos tradicionais não são suficientes para representá-la (KHOROSHEV, MEREKALOVA \& ALESCHENKO, 2007; PUZACHENKO, 2007; CHERKASHIN, 2008).

Percebe-se que os modelos tradicionais são eficazes para compreensão da gênese da paisagem, mas não para compreensão 
CAVALCANTI, L.C.S. \& CORRÊA, A.C.B. Problemas de hierarquização...

do seu funcionamento, que depende bastante das escalas espaciais e temporais escolhidas para observação do fenômeno e da ideia de funcionalidade. Neste sentido, o próprio modelo hierárquico Fácies, Subtrato, Trato, Localidade, Paisagem (e modelos similares) vem sendo questionada (FROLOVA, 2006; KHOROSHEV, MEREKALOVA \& ALESCHENKO, 2007).

Dentre as alternativas metodológicas, tem-se procurado evitar níveis de organização a priori, buscando-se detectar as relações de hierarquia a partir das relações funcionais entre os componentes de vários níveis (SYSUEV, 2004; KHOROSHEV, MEREKALOVA \& ALESCHENKO, 2007; KHOROSHEV \& ALESCHENKO, 2008).

Buscando considerar a funcionalidade da paisagem, alguns modelos alternativos vêm assumindo o componente relevo como principal fator que determina o estado de outros geocomponentes subordinados, visto que, a partir de seus atributos (aspecto, declividade, curvatura, altitude, etc.), condiciona a direção, intensidade e sentido de grande parte dos fluxos de substâncias geográficas (SYSUEV, 2004; KHOROSHEV, MEREKALOVA \& ALESCHENKO, 2007), enquanto que a cobertura vegetal é tida como o principal indicador das fronteiras entre tipos de padrões de paisagens e, consequentemente, entre tipos de processos (MARCEAU, 1999; WU \& DAVID, 2002; KHOROSHEV, MEREKALOVA \& ALESCHENKO, 2007).

Khoroshev \& Aleschenko (2008) levantaram alguns aspectos que devem ser considerados quanto ao estudo funcional da hierarquia de paisagens e sua aplicação para o planejamento territorial:

1. A diferença entre o nível do funcionamento da paisagem (onde são coletados os dados) e aquele da tomada de decisão;

2. A consideração de níveis superiores e inferiores ao nível estudado;

3. A consideração da hierarquia (espacial e temporal) particular de cada geocomponente; 
CAVALCANTI, L.C.S. \& CORRÊA, A.C.B. Problemas de hierarquização...

4. A auto-similaridade entre os níveis de organização, que pode revelar aspectos da hierarquia;

5. A discriminação do papel de cada fator em diferentes níveis hierárquicos, considerando a variabilidade das variáveis analisadas.

Khoroshev, Merekalova \& Aleschenko (2007) e Khoroshev \& Aleschenko (2008) utilizaram uma série de técnicas computacionais (equações de multi-regressão, medidas informacionais de conectividade, coeficientes de correlação nãoparamétrica e Determinante Jacobiana) para detectar as relações de dependência dos parâmetros vegetais sensíveis aos higrótopos, que variam com o relevo. Contudo, seus estudos basearam-se em aproximação multiescalar indireta, definindo níveis hierárquicos arbitrários para aplicação das técnicas escolhidas: os autores avaliam as relações em terrenos definidos com base em pixels reamostrados de 400 metros com base em imagem Landsat 7.

Geógrafos canadenses (HAY \& MARCEAU, 2004) propuseram um complexo método de análise multiescalar, denominado Multiscale Object-Specific Analysis (MOSA). A proposta de Hay \& Marceau é uma abordagem upscaling baseada nas respostas espectrais dos objetos imageados, constituindo uma aproximação multiescalar direta. Esta técnica baseia-se no uso de imagens de satélite de alta-resolução, a partir das quais se aglutinam pixels com características semelhantes em objetos. Estes objetos são expandidos por uma função ponderada com base em sua influência na cena, criando um contexto hierárquico superior (sistemas antecedentes), este novo contexto é segmentado, dando origem a novos objetos. O problema deste método é que ele não necessariamente reflete a ideia de fluxos na paisagem, fluxos de encosta, suprimindo a compreensão do contingenciamento de unidades de paisagem.

Puzachenko (1997 apud. PUZACHENKO, 2007) propõe uma aproximação multiescalar direta com base na análise fractal e multifractal de padrões espaciais. Contudo, o modelo usa geocomponentes baseados na emissão de calor obtidos por 
CAVALCANTI, L.C.S. \& CORRÊA, A.C.B. Problemas de hierarquização...

Sensoriamento Remoto, apresentando relações de dependência da paisagem, sem, contudo, apresentar uma consideração global do funcionamento da paisagem. Segundo o mesmo autor, o problema de escolha das variáveis é o maior empecilho na modelagem de geossistemas (PUZACHENKO, 2007).

Sysuev (2004) e Sysuev \& Aleschenko (2007) propõem o uso de trabalhos de campo para escolha das variáveis a serem utilizadas numa classificação automática com base no algoritmo $k$ means que, inicialmente define $k$ classes a priori e então aglomera classe por classe em um processo iterativo usando a distância euclidiana mínima, o objetivo é minimizar a variabilidade no interior dos agrupamentos e maximizar entre agrupamentos (SYSUEV \& ALESCHENKO, 2007). A vantagem desta técnica é que ela evita níveis a priori e baseia as classificações em dados reais. Os resultados indicaram boas relações entre os limites dos geossistemas definidos em campo com os limites obtidos pela classificação automática. O problema desta abordagem é que ela não pode ser classificada como uma aproximação multiescalar, visto que os autores definem apenas um nível, geralmente urochishches (Tratos) ou mestnosts (Localidades).

Percebe-se que o enfrentamento da questão hierárquica é complexo. Os modelos tradicionais concentram-se em aspectos genéticos, não sendo usuais as aproximações do ponto de vista da análise funcional, esta carece de uma seleção coerente das variáveis que serão utilizadas para modelagem. Outrossim, o papel do monitoramento ambiental parece essencial. Os modelos alternativos se baseiam em Sensoriamento Remoto, SIG e/ou Programação na tentativa de detectar a funcionalidade da paisagem, contudo ainda sofrem com a escolha das variáveis e com a dificuldade em implementar aproximações multiescalares diretas. 
CAVALCANTI, L.C.S. \& CORRÊA, A.C.B. Problemas de hierarquização...

\section{Conclusões}

A partir do que foi exposto verifica-se que não existe consenso em relação ao modelo ideal de geocomponentes para o estudo da paisagem, além do que não existe consenso acerca dos níveis de organização que compõem a hierarquia da paisagem. A própria existência de um modelo hierárquico com níveis prédefinidos vem sendo colocada em xeque, todavia uma solução ainda não foi encontrada. Em grande parte dos modelos de representação da hierarquia a representação da dinâmica da paisagem vem sendo apontada como fundamental para a compreensão da hierarquia dos geossistemas.

\section{Agradecimentos}

Ao CNPq pela concessão da bolsa de Mestrado.

\section{Referências bibliográficas}

AB'SÁBER, A.N. Domínios de Natureza no Brasil: potencialidades paisagísticas. 6 Ed. São Paulo: Ateliê Editorial. 2011. 159p.

AHL, V. \& ALLEN, T.F.H. Hierarchy Theory. A vision, vocabulary and epistemology. New York. Columbia University Press. 1996. 206p.

BERTRAND, G. Paisagem e Geografia Física global: um esboço metodológico. Caderno de Ciências da Terra. N.13. São Paulo. IGUSP. $1972.27 \mathrm{p}$.

BERUCHASHVILI, N.L. Geographical perspective on training of students in sustainable development in Georgia In: ROBERTSON, M. (org.) Sustainable futures: teaching and learning: a case approach. Camberwell, Victoria. Acer Press. 2007. p.209-224. 
CAVALCANTI, L.C.S. \& CORRÊA, A.C.B. Problemas de hierarquização...

CHERKASHIN, A.K. Geographical systemology: formation rules for system ontologies. Geography and Natural Resources. 2008. V.29. n.2. p.110-115.

FROLOVA, M. Desde el concepto de paisaje a la Teoría de geossistema en la Geografía Rusa: ¿hacia una aproximacíon global del médio ambiente? Ería. N.70. 2006. p.225-235.

HAY, G. J., \& MARCEAU, D. J. 2004. Multiscale Object-Specific Analysis (MOSA): An integrative approach for multiscale landscape analysis. In: De Jong S. M. \& Van Der Meer F. D. (Eds). Remote Sensing and Digital Image Processing. Volume 4. Chapter 3. Kluwer Academics. (In review).

ISACHENKO, A.G. Principles of Landscape Science and Physical-geographic Regionalization. Melbourne. Melbourne University Press. 1973. 320p.

ISACHENKO, G.A. Long-term conditions of taiga landscapes of European Russia. In: DYAKONOV, K.N., KASIMOV, N.S., KHOROSHEV, A.V., KUSHLIN, A.V. Landscape analysis for sustainable development: theory and applications of Landscape Science in Russia. Alexplublishers: Moscow. 2007. p.144-155.

KHOROSHEV, A.V. \& ALESCHENKO, G.M. Methods to identify geosystems with a commonalty of intercomponent Relationships. Geography and Natural Resources. 2008. V.29. n.3. p.267-272.

KHOROSHEV, A.V. \& MEREKALOVA, K.A. Uncertainty of relations between landscape components - a tool for modeling evolution of spatial pattern. Ekológia. 25:122-130, 2006.

KHOROSHEV, A.V. MEREKALOVA, K.A. \& ALESCHENKO, G.M. Multiscale organization of intercomponent relations in landscape. In: DYAKONOV, K.N., KASIMOV, N.S., KHOROSHEV, A.V., KUSHLIN, A.V. Landscape analysis for 
CAVALCANTI, L.C.S. \& CORRÊA, A.C.B. Problemas de hierarquização... sustainable development: theory and applications of Landscape Science in Russia. Moscow: Alexplublishers. 2007. p.93-103.

KRAUKLIS, A.A. Problemas de Ciência da Paisagem Experimental. Novosibirsk. Nauka. 233p. (em russo).

KRUHLOV, I. The structure of the urban landscape. Universitas Ostraviensis. Acta Facultatis Rerum Naturalium. Geographia Geologia, 181/7: 71-89. 1999. 12p.

KRUHLOV, I. \& BOZHUK, T. Geoecological information system of the Ukrainian Maramorosh. In: WIDACKI, W., BYTNEROWICZ, A. \& RIEBAU, A. A message from the Tatra. Geographical information systems and Remote Sensing in mountain environmental research. Jagiellonian University Press. 2004. p.173-182.

LEVIN, S.A. The problem of pattern and scale in ecology. Ecology. 1992. n.73, p.1943-1967.

MAMAY, I.I. Landscape Science in Russia in the early XXI century: state and methodological problems. In: DYAKONOV, K.N., KASIMOV, N.S., KHOROSHEV, A.V., KUSHLIN, A.V. Landscape Analysis for sustainable development: theory and applications of landscape science in Russia. Moscou: Alexplublishers, 2007. p.21-28.

MARCEAU, D.J. The scale issue in social and natural sciences. Canadian Journal of Remote Sensing. 1999. V.25, N.4, p.347356.

MELO, D.R. Evolução das Veredas sob Impactos Ambientais nos Geossistemas Planaltos de Buritizeiros/MG. (Tese de Doutorado em Geografia). Belo Horizonte: UFMG. 2008. 353p. 
CAVALCANTI, L.C.S. \& CORRÊA, A.C.B. Problemas de hierarquização... MONTEIRO, C. A. F. Teoria e Clima Urbano. (Tese de Livre Docência). São Paulo: USP, 1976. 181p.

PUYEO, Y., ALADOS, C.L., MAESTRO, M., KOMAC, B. Gypsophile vegetation patterns under a range of soil properties induced by topographical position. Plant Ecology. N.189. 2007. p.301-311.

PUZACHENKO, Y.G. Landscape as a dynamical and thermostatic system. In: DYAKONOV, K.N., KASIMOV, N.S., KHOROSHEV, A.V., KUSHLIN, A.V. Landscape Analysis for sustainable development: theory and applications of landscape science in Russia. Moscou: Alexplublishers, 2007. p.29-41.

SIMON, H.A. The architecture of complexity. Proceedings of the American Philosophical Society. 1962. n.106. p.467-482.

SOARES FILHO, B.S. Modelagem da Dinâmica de Paisagem de uma região de fronteira de colonização amazônica. Tese (Doutorado em Engenharia). São Paulo. 1998. 299p.

SOCHAVA, V.B. O estudo de geossistemas. Métodos em questão, n.16, IGUSP. São Paulo, 1977. 51 p.

SOLON, J., DEGÓRSKI, M., ROO-ZIELIŃSKA, E. Vegetation response to a topographical-soil gradient. Catena. N.71. 2007. p.309-320.

SOLNETCEV, N.A. The natural geographic landscape and some of its general rules. In: WIENS, J.A.; MOSS, M.R.; TURNER, M.G.; MLADENOFF, D.J. Foundation papers in Landscape Ecology. Columbia: Columbia University Press. 2006. p.19-27.

SYSUEV, V.V. Modeling geosystem differentiation. Série Geographica. 31: 340-349. 2004. 
CAVALCANTI, L.C.S. \& CORRÊA, A.C.B. Problemas de hierarquização...

SYSUEV, V.V \& ALESCHENKO, G.M., Modeling landscapes: toward synthesis based on geophysical parameters. In: DYAKONOV, K.N., KASIMOV, N.S., KHOROSHEV, A.V., KUSHLIN, A.V. Landscape Analysis for sustainable development: theory and applications of landscape science in Russia. Moscou, Alexplublishers, 2007. p. 58-75.

WU, J. \& DAVID, J.L. A spatially explicit hierarchical approach to modeling complex ecological systems: theory and applications. Ecological Modelling. 2002. n.153. p. 7-26.

XU, X.L., MA, K.M., FU, B.J., SONG, C.J., LIU, W. Relationships between vegetation and soil and topography in a dry warm river valley, SW China. Catena. N.75. 2008. p.138-145.

ZONNEVELD, I.S. Land Unit: a fundamental concept in Landscape Ecology, and its applications. Landscape Ecology. V.3. n.2. 1989. p.67-86.

ZUCHKOVA, V.K. \& RAKOVSKAIA, E.M. Métodos de Pesquisa em Geografia Física Integrada. Moscou: Academia. 2004. 368p. (em russo).

Recebido em abril de 2012 Aceito em abril 2013 